\title{
Investigation of the effects caused by applying voltage in Layer-by-Layer self-assembly method
}

\author{
Y. Omura ${ }^{1}$, K.H. Kyung ${ }^{1}$, S.H. Kim ${ }^{2}$, and S. Shiratori ${ }^{1}$ \\ ${ }^{1}$ Graduate School of Science and Technology, Keio Univ., 3-14-1 Hiyoshi, Kohoku-ku, Yokohama-shi, kanagawa-ken 223- \\ 8522, Japan \\ ${ }^{2}$ Department of Ceramic Engineering, Kangnung-wonju National Univ., 123 Jibyeon-dong, Kangwon-do 210-702, Korea
}

\begin{abstract}
Recently, Layer-by-Layer (LbL) self-assembly method under applied voltage (voltage-applied LbL) attracts great attention. It is reported that the method enables more abundant film adsorption than conventional LbL method. However, a small proportion of experimental results about adsorption of polyelectrolytes by voltage-applied LbL have been reported. In this study, voltage-applied LbL method using weakly charged polyelectrolytes was examined. Poly (allylamine hydrochloride) (PAH) and Poly (ethylene imine) (PEI) as cationic solutions and Poly (acrylic acid) (PAA) as anionic solution were chosen. The $\mathrm{pH}$ of solutions was adjusted to several conditions and film of PAH/PAA and film of PEI/PAA were fabricated by voltage-applied LbL method. Change of adsorption behavior and film morphology by applying voltage depended on $\mathrm{pH}$ condition of solutions. When $\mathrm{pH}$ of PAH/PAA solutions was 3.9/3.8, respectively, the film adsorption was accelerated by applying voltage. Moreover, in this condition, the surface morphology remarkably changes and texture structure appears by applying voltage. Consequently, it was found that applying voltage in LbL method was effective in controlling film adsorption and the surface nano structure.
\end{abstract}

\section{Introduction}

LbL method is a promising technique for fabricating uniform thin films composed of polyelectrolytes. They are suitable for various applications, such as optical films [1], antithrombotic films [2], superhydrophobic surfaces [3], separation films [4], biosensors [5], chemical sensors [6]. The method is eco-friendly in using aqueous medium and enables controlling thickness in nanometer scale under ordinary temperature and normal pressure [7]. Though many advantages of LbL method, the adsorption process takes a long adsorption time because it depends on electrostatic adsorption of oppositely charged polyelectrolytes [8]. To solve this problem, spray LbL method, in which solutions are sprayed directly onto substrates, has been developed [9]. Moreover, applying external force to the LbL process has been attempted to enhance adsorption. In recent study, LbL method under applied voltage attracts great attention. It is reported that the method enables more abundant film adsorption than conventional LbL method [10,11]. However, a small proportion of experimental results about adsorption of polyelectrolytes by voltage-applied LbL have been reported.

The characteristics of film of polyelectrolytes depend on the intrinsic properties of polyelectrolytes, such as the polymer chain conformation, the molecular weight and the attached functional group. Moreover, they can also be changed by adding salts to the solutions or adjusting the $\mathrm{pH}$ of the solutions [12, 13]. In particular, weakly charged polyelectrolytes are subject to be influenced by $\mathrm{pH}$ condition. The polymer chain conformation changes because the degree of ionization of the polyelectrolytes varies. Therefore, the film thickness can easily be changed by adjusting the $\mathrm{pH}$ of solutions [14]. In addition, it is also reported that surface morphology can be varied by adjusting it. In film of PAH/PAA and film of PEI/PAA, texture structure appears at specific $\mathrm{pH}$ conditions [15].

In this study, film of PAH/PAA and film of PEI/PAA were fabricated by voltage-applied LbL method. And also the $\mathrm{pH}$ of polyelectrolyte solutions was adjusted to several conditions. Then, we investigate of the effects of applied voltage on adsorption behavior and film morphology in fabricating film of PAH/PAA and film of PEI/PAA.

\section{Materials and Methods}

\subsection{Materials}

Poly (diallyldimethyl ammonium chloride) (PDDA, Mw 200,000-350,000) and Poly (sodium 4-styrenesulfonate) 
(PSS, Mw 70,000) and Poly (acrylic acid) (PAA, Mw $\sim 100,000)$ were obtained from Aldrich. Poly (allylamine hydrochloride) (PAH, Mw 120,000-200,000) and Poly (ethylene imine) (PEI, Mw 70,000) were obtained from Wako. PDDA, PAH and PEI were used as cation and PSS and PAA were anion. $10 \mathrm{mM}$ solutions in deionized water were prepared, respectively. PDDA and PSS solutions were unadjusted. PAH, PAA and PEI solutions were adjusted to several $\mathrm{pH}$ conditions by addition of diluted solutions of hydrochloric acid and sodium hydroxide.

\subsection{Preparation of the substrate}

Quartz Crystal Microbalance (QCM) was used as the substrate and cleaned by sonication in potassium hydroxide $(\mathrm{KOH})$ for $5 \mathrm{~min}$ and in deionized water for 2 min three times. Subsequently, QCM was blown-dried and (PDDA/PSS) $)_{5}$ film was fabricated on QCM as buffer layer. The role of buffer layer is to decrease the surface roughness and increase negative electrical charges on the surface. Deposition time in solutions was $5 \mathrm{~min}$, respectively. After each deposition step QCM was rinsed with ultrapure water for 1 min 3 times.

\subsection{LbL assembly of polyelectrolytes under applying voltages}

Cationic and anionic solutions were put in containers, respectively. QCM as the substrate (working electrode) and Pt as the counter electrode were parallel set up in the containers and the distance between the electrodes is 15 mm. $\mathrm{Ag} / \mathrm{AgCl}_{2}$ was used as the reference electrode. Three electrodes were connected to a DC power supply. A voltage of $0.5 \mathrm{~V}, 1.0 \mathrm{~V}$ or $1.5 \mathrm{~V}$ was applied between the substrate and counter electrode during the deposition step. The substrate was the anode in cationic solution and the anode in anionic solution. After each deposition step, substrate was rinsed with ultrapure water $1 \mathrm{~min}$. These steps were repeated 5 times and films of $(\mathrm{PAH} / \mathrm{PAA})_{5}$ and $(\mathrm{PEI} / \mathrm{PAA})_{5}$ were fabricated.

\subsection{Characterization}

The amount of adsorption of after each deposition step was measured by QCM. The surface morphology was observed by Scanning Electron Microscope (SEM) and the surface roughness was investigated by atomic force microscopy (AFM).

\section{Results and Discussion}

\subsection{Amount of adsorption}

The amount of adsorption of after each deposition step was measured by QCM. The amount of adsorption of $(\mathrm{PAH} / \mathrm{PAA})_{5}$ films fabricated in several $\mathrm{pH}$ conditions is shown in Fig.1. When $\mathrm{pH}$ of PAH/PAA solutions was 3.9/3.8, 5.5/9.0 and 7.0/7.0, respectively, the amount of adsorption was increased by applying voltage.

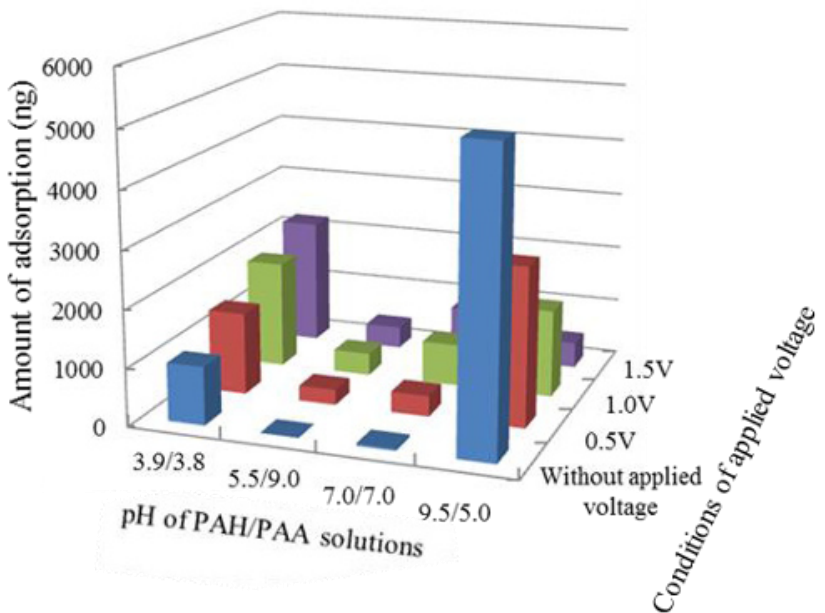

Fig.1. Amount of adsorption of (PAH/PAA $)_{5}$ films fabricated without applied voltage and under applied voltage of $0.5 \mathrm{~V}$,

$1.0 \mathrm{~V}, 1.5 \mathrm{~V}$. ( pH: 3.9/3.8, 5.5/9.0, 7.0/7.0, 9.5/5.0 )

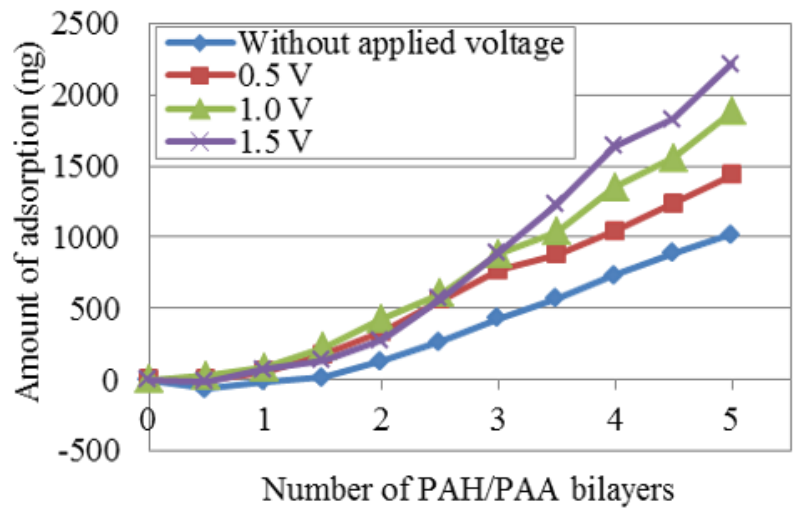

Fig.2. Adsorption behavior of (pH3.9 PAH/pH3.8 PAA $)_{5}$ films fabricated without applied voltage and under applied voltage of $0.5 \mathrm{~V}, 1.0 \mathrm{~V}, 1.5 \mathrm{~V}$.

However, when $\mathrm{pH}$ of PAH/PAA solutions was 9.5/5.0, the amount of adsorption was decreased by applying voltage. Then, adsorption behavior of ( $\mathrm{pH} 3.9 \mathrm{PAH} / \mathrm{pH} 3.8$ $\mathrm{PAA})_{5}$ films fabricated without applied voltage or under applied voltage was shown in Fig.2. It was found that the film adsorption was drastically enhanced by the increase in applied voltage. Indeed, the amount of adsorption at $\mathrm{pH} 9.5 / 5.0$ without applied voltage is the highest. However, applying voltage enables the precise control of the amount of adsorption. Moreover, in the case of the $\mathrm{pH}$ conditions of PAH/PAA solution, in which the amount of adsorption without applied voltage was practically nought, we were able to promote film deposition by applying voltage. The change of adsorption behavior of $(\mathrm{PEI} / \mathrm{PAA})_{5}$ by applying voltage was similar to that of $(\mathrm{PAH} / \mathrm{PAA})_{5}$ films.

\subsection{Surface morphology}

The surface morphology of (PAH/PAA $)_{5}$ films fabricated without applied voltage or under applied voltage of $0.5 \mathrm{~V}$, $1.0 \mathrm{~V}, 1.5 \mathrm{~V}$ was observed by scanning electron microscope (SEM), shown in Fig. 3. When $\mathrm{pH}$ of PAH/PAA solutions were 3.9/3.8, respectively, the surface morphology remarkably changed and texture structure 


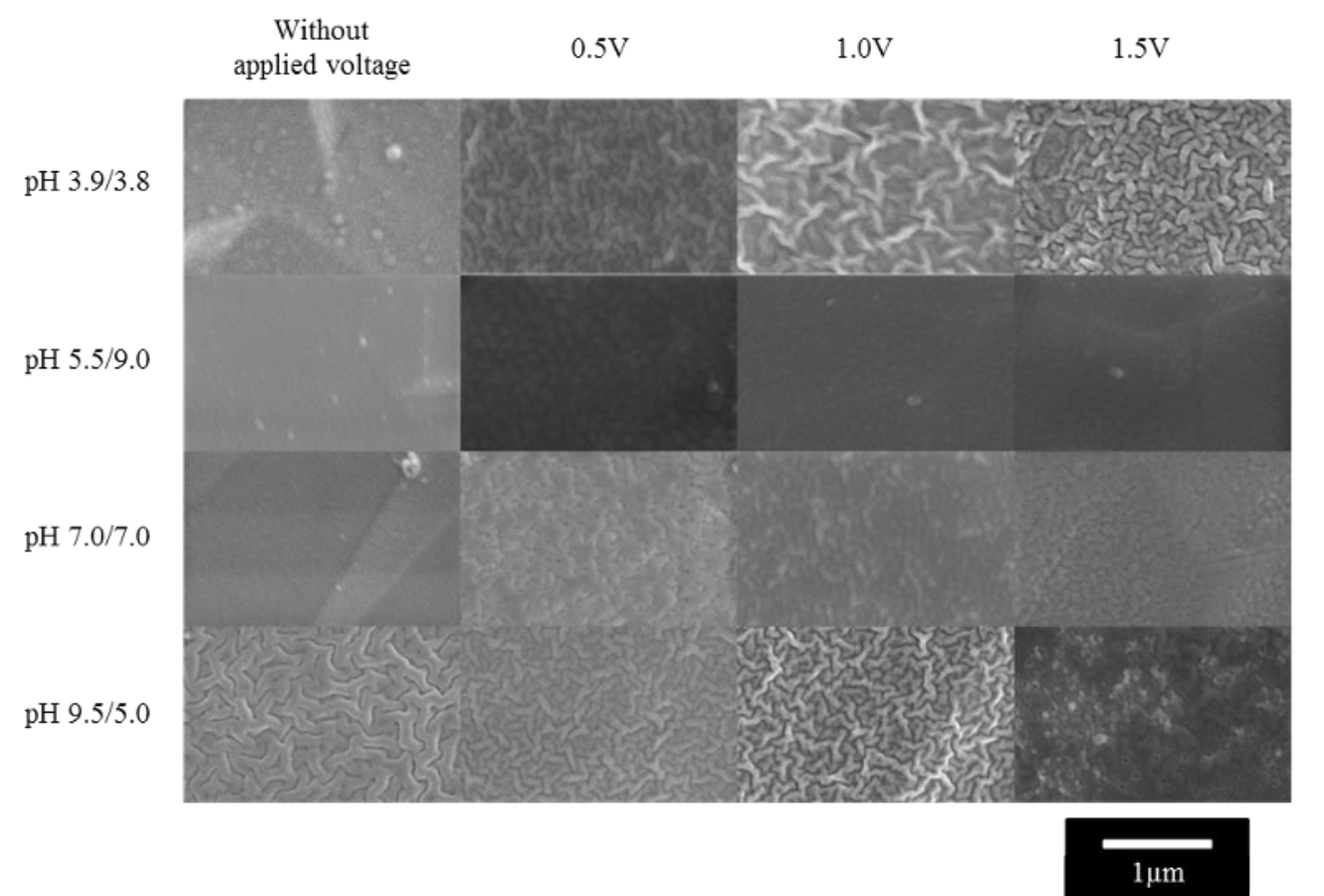

Fig.3. Surface morphology of (PAH/PAA $)_{5}$ films fabricated without applied voltage and under applied voltage of $0.5 \mathrm{~V}, 1.0 \mathrm{~V}, 1.5 \mathrm{~V}$. ( $\mathrm{pH}: 3.9 / 3.8,5.5 / 9.0,7.0 / 7.0,9.5 / 5.0)$

Table.1. RMS roughness of (PAH/PAA $)_{5}$ films fabricated without applied voltage or under applied voltage of $0.5 \mathrm{~V}$ ( $\mathrm{pH}: 3.9 / 3.8,7.0 / 7.0,9.5 / 5.0)$ or $1.0 \mathrm{~V}(\mathrm{pH}: 5.5 / 9.0)$

\begin{tabular}{|c|c|c|}
\hline \multirow{2}{*}{$\begin{array}{c}\mathrm{pH} \text { conditions } \\
\text { of PAH/PAA }\end{array}$} & \multicolumn{2}{|c|}{ RMS (nm) } \\
\cline { 2 - 3 } & Without applied voltage & under applied voltage \\
\hline $3.9 / 3.8$ & 6.3 & 29.3 \\
\hline $5.5 / 9.0$ & 4.6 & 13.7 \\
\hline $7.0 / 7.0$ & 8.6 & 15.8 \\
\hline $9.5 / 5.0$ & 28.7 & 13.6 \\
\hline
\end{tabular}

appeared by applying voltage. Texture structure also appeared on $(\mathrm{pH} 9.5 \mathrm{PAH} / \mathrm{pH} 5.0 \mathrm{PAA})_{5}$ films inspite of presence of applied voltage. However, size of the structure was changed by applying voltage. RMS roughness of $(\mathrm{PAH} / \mathrm{PAA})_{5}$ films fabricated without applied voltage or under applied voltage was observed by atomic force microscope (AFM), shown in Table.1. Applied voltage is $0.5 \mathrm{~V}$ for $\mathrm{pH} 3.9 / 3.8,7.0 / 7.0,9.5 / 5.0$ and $1.0 \mathrm{~V}$ for $\mathrm{pH} 5.5 / 9.0$. In the case of $\mathrm{pH} 3.9 / 3.8,7.0 / 7.0$, $5.5 / 9.0$, the amount of adsorption was increased by applied voltage (Fig.1) Therefore, RMS roughness increased by applying voltage. Moreover, considering the results in Fig. 3 and Table.1, it is thought that nano scale structure, which was due to the change of polymer chain conformation, was controlled by applying voltage. Therefore, we consider that the change of the surface structure was not due to the change of polymer chain conformation of adsorbed layer with the $\mathrm{pH}$ of the solution of the next adsorbed layer.

\subsection{The relationship between amount of adsorption and applied voltage}

The change of adsorption behavior by applying voltage can be discussed using two LbL adsorption process model shown in Fig.4. In the case of fabricating films without applied voltage, in initial state, there are hydroxyl groups on the substrate. PAH is deposited on the substrate by electrostatic adsorption of them. Then, PAA is deposited and adsorption step is repeated. On the other hand, in the case of fabricating films under applied voltage, there are more electrical charges on the substrate. Therefore, polyelectrolyte adsorption is promoted. Moreover, the effects by applying voltage can be discussed by formula (1). It is developed by Stokes's formula. Migration speed of molecules $v$ is calculated from electrical charges of molecules $Q$ and electric field $E$, radius of molecules $r$ and viscosity of solutions $\eta$.

$v=\frac{Q E}{6 \pi r \eta}$

Formula (1) shows that migration speed $v$ is in proportion to electric field $E$ if polymers are particles. Because electric field increases with increase in the applied 


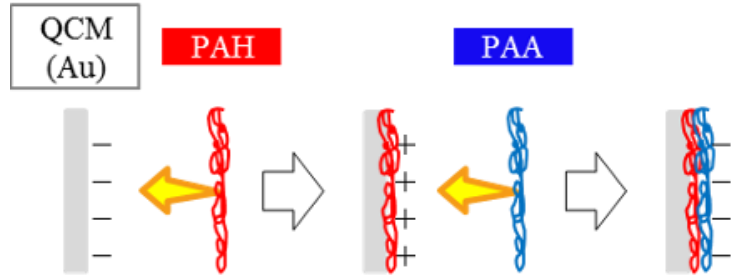

(a) Without applied voltage.

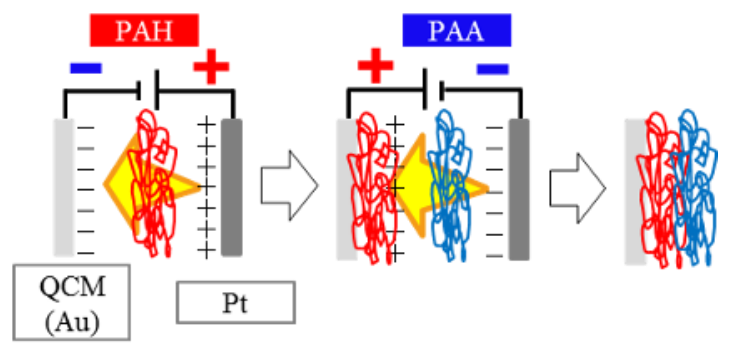

(b) Under applied voltage.

Fig.4. Comparison of two LbL adsorption process.

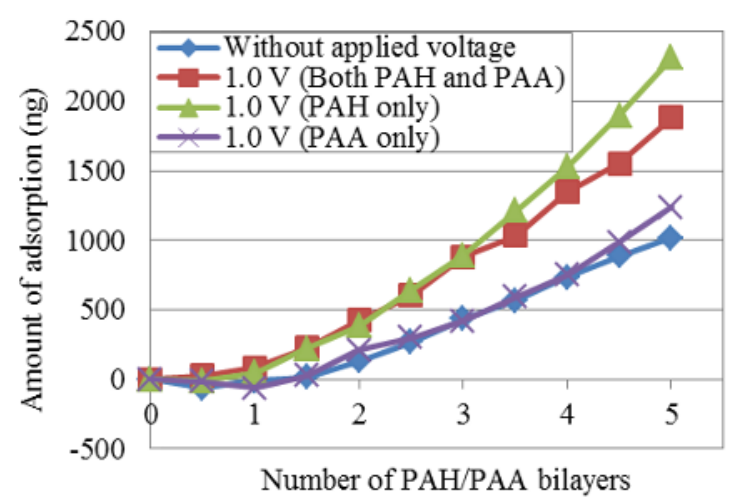

Fig.5. Adsorption behavior of (pH3.9 PAH/pH3.8 PAA $)_{5}$ films fabricated without applied voltage and under applied voltage of 1.0V. (Both PAH and PAA, PAH only, PAA only)

voltage, the migration of polyelectrolytes is accelerated by applying voltage.

However, the amount of adsorption was decreased by applying voltage when $\mathrm{pH}$ of $\mathrm{PAH} / \mathrm{PAA}$ solution was 9.5/5.0. Then, the relationship between change of amount of adsorption by applying voltage and combination of polymer chain conformation was investigated. The polymer chain conformation of weakly charged polyelectrolyte is changed by the degree of ionization which changes with $\mathrm{pH}$ conditions [16]. Then, considering the $\mathrm{pKa}$ of polyelectrolytes, the $\mathrm{pKa}$ of PAH/PAA is about 8.8/6.5, respectively [17]. When the degree of ionization of polyelectrolyte is lower (PAH at high $\mathrm{pH}$ and PAA at low $\mathrm{pH}$ ), the polymer chain conformation is loop or tail structure. On the other hand, when the degree of ionization of polyelectrolyte is higher (PAH at low $\mathrm{pH}$ and PAA at high $\mathrm{pH}$ ), the polymer chain conformation is train structure. Therefore, when $\mathrm{pH}$ of PAH/PAA solution was 9.5/5.0, polymer chain conformations of both $\mathrm{PAH}$ and PAA are loop or tail structure. Then, (pH3.9 PAH/pH3.8 PAA $)_{5}$ films were fabricated under applying voltage in adsorption step of either PAH or PAA and the adsorption behavior is shown in Fig.5. In this condition, polymer chain conformations of PAH and PAA are train and loop or tail. Amount of adsorption is abounding in order of films fabricated under applying voltage in adsorption step of PAH, both PAH and PAA, PAA, neither PAH or PAA. That is to say, it is thought that the amount of adsorption increase in the case that the voltage is applied during adsorption of polyelectrolyte the polymer chain conformation of which is train structure.

\section{Conclusion}

In this study, film of PAH/PAA and film of PEI/PAA were fabricated by voltage-applied LbL method. Change of adsorption behavior and film morphology by applying voltage depended on $\mathrm{pH}$ condition of solutions. When $\mathrm{pH}$ of PAH/PAA solutions were 3.9/3.8, respectively, the film adsorption was accelerated and texture structure was observed. Consequently, it was found that applying voltage in LbL method was effective in controlling film adsorption and the surface morphology.

\section{References}

1. Z. A. Khan, R. Kumar, W. S. Mohammed, G. L. Hornyak, J. Dutta, J. Mater. Sci., 46, 21, 6877-6882 (2011)

2. W. Y. Cai, J. F. Wu, C. W. Xi, A. J. Ashe, M. E. Meyerhoff, Biomaterials, 32, 31, 7774-7784 (2011)

3. L. Zhang, Y. Li, J. Sun, J. Shen, J. Colloid Interface Sci., 319, 1, 302-308 (2008)

4. G. Zhang, Z. Ruan, S. Ji, Z. Liu, Langmuir, 26, 7, 4782-4789 (2010)

5. C. A. Constantine, K. M. Gattas-Asfura, S. V. Mello, G. Crespo, V. Rastogi, T. C. Cheng, J. J. DeFrank, R. M. Leblanc, Langmuir, 19, 23, 9863-9867 (2003)

6. G. Xie, P. Sun, X. Yan, X. Du, Y. Jiang, Sens. Actuators B Chem., 145, 1, 373-377 (2010)

7. G. Decher, Science, 277, 5330, 1232-1237 (1997)

8. M. Bruening, D. Dotzauer, Nat. Mat., 8, 6, 449-450 (2009)

9. A. Izquierdo, S. S. Ono, J. C. Voegel, P. Schaaf, G. Decher, Langmuir, 21, 16, 7558-7567 (2005)

10. Y. H. Ko, Y. H. Kim, J. H. Park, P. J. Yoo, K. T. Nam, Macromolecules, 44, 8, 2866-2872 (2011)

11. G. Zhang, L. Dai, L. Zhang, S. Ji, Langmuir, 27, 6, 2093-2098 (2011)

12. J. B. Schlenoff, H. Ly, M. Li, J. Am. Chem. Soc., 120, 30, 7626-7634 (1998)

13. D. Yoo, S. S. Shiratori, M. F. Rubner, Macromolecules, 31, 13, 4309-4318 (1998)

14. S. S. Shiratori, M. F. Rubner, Macromolecules, 33, 11, 4213-4219 (2000)

15. S. Fujita, S. Shiratori, Nanotechnology, 16, 9, $1821-$ 1827 (2005)

16. S. Beltran, H. H. Hooper, H. W. Blanch, J. M. Prausnitz, Macromolecules, 24, 11, 3178-3184 (1991)

17. J. Choi, M. F. Rubner, Macromolecules, 38, 1, 116124 (2005) 